\title{
EFFECT OF BROMELAIN AND TRYPSIN ON MICROLEAKAGE OF ETCH-AND-RINSE ADHESIVE SYSTEMS
}

\author{
Shahin Kasraie', Ebrahim Yarmohammadi², Maryam Farhadian ${ }^{3}$, Mona Malek $^{4}$ \\ 'Department of Restorative Dentistry, Dental School of Shahid Beheshty University of Medical Science, Tehran, Iran \\ 2Department of Restorative Dentistry, Dental School of Hamedan University of Medical Sciences, Iran \\ ${ }^{3}$ Department of Biostatistics, School of Public Health and Research Center for Health Sciences, Hamadan University of Medical Sciences, Iran \\ ${ }^{4}$ Dental Clinic of AmirAlam Hospital, Tehran University of Medical Sciences, Tehran, Iran
}

\begin{abstract}
INTRODUCTION: The removal of collagen by chemical methods can effectively decrease the marginal microleakage of composites used in restorative dentistry.

Овјестіves: To present research focused on the assessment of the impact of surface treatment of dentin with $5 \%$ bromelain and $5 \%$ trypsin on the microleakage of composites. To present research focused on the assessment of the impact of surface treatment of dentin with $5 \%$ bromelain and $5 \%$ trypsin on the microleakage of composites.

MATERIAL AND METHODS: In the lingual and buccal surfaces of 60 extracted premolars, class V cavities were prepared in standard condition. The test cases were divided into three groups $(n=40)$. In the control group, 37\% phosphoric acid was employed for etching the cavities. In one of the groups, Adper Single Bond was utilised in the wall of cavity, while OptiBond Solo was applied in the other group. Afterwards, the samples were restored by employing Z250 composite. 5\% bromelain and 5\% trypsin were used in the test groups. The samples were then immersed in $2 \%$ fuchsine solution to analyse the marginal microleakage.

RESULTS: No significant difference was observed in the occlusal marginal microleakage of study groups ( $p=0.43)$, but the difference in microleakage of cervical margins was significant $(p=0.000)$.

Conclusions: Pretreatment of cavity by using $5 \%$ bromelain in the etched dentin surface showed no significant impact on microleakage. However, using 5\% trypsin after etching has a negative effect on the microleakage at the cervical margins.
\end{abstract}

KEY WORDS: trypsin, etch-and-rinse adhesive system, microleakage.

J Stoma 2020; 73, 4: 183-192

DOI: https://doi.org/10.5114/jos.2020.98314

\section{INTRODUCTION}

The introduction of dentin adhesive systems and understanding their interactions with dentin revolutionised restorative dentistry [1]. The nano and microleakage level of the materials used in dental treatment is the main concern of various studies. Some degrees of marginal microleakage have been reported in composite restorations bonded with different dentin bonding agents $[2,3]$. In the recent years, despite advances in dental adhesives, the hybrid layer still remains the most fragile area in terms of bond strength [4]. The hybrid layer made by etch-and-rinse adhesives degrades over time. This degradation is characterised by the loss of collagen fibres and destruction of the hybrid layer followed

JOURNAL OF STOMATOLOGY CZASOPISMO STOMATOLOGICZNE

AdDress For CORRESPondence: Dr. Mona Malek, Dental Clinic of AmirAlam Hospital, Tehran University of Medical Sciences, Iran, e-mail: mona_mlk@yahoo.com 


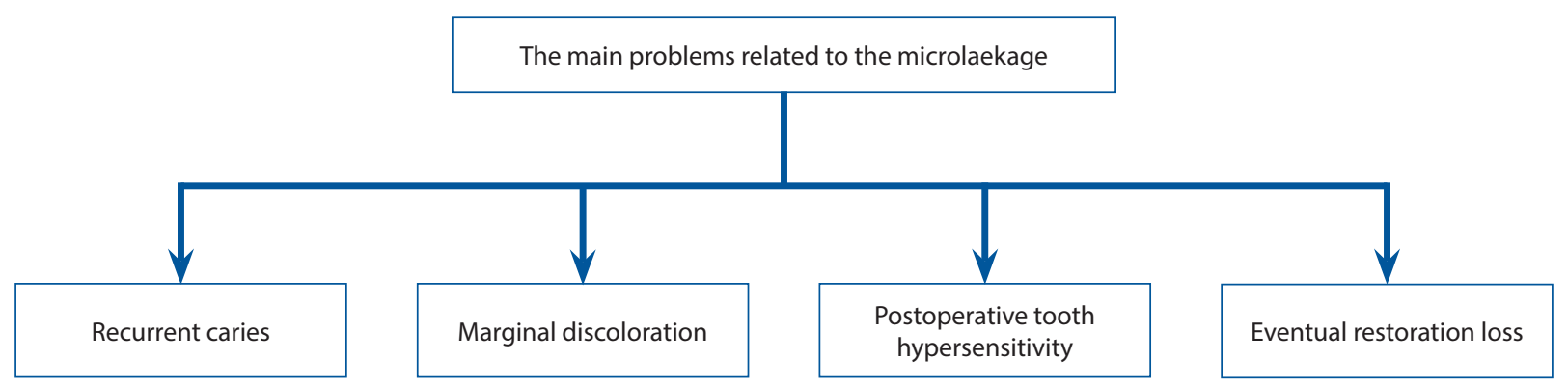

FIGURE 1. Some of the main problems due to microleakage

by water sorption. Incomplete formation of the hybrid layer and subsequent microleakage cause various problems, as shown in Figure 1 [5].

Acid etching of the tooth surfaces demineralises 5-10 $\mu \mathrm{m}$ of the superficial dentin, and a relatively demineralised area of dentin is located close to this region $[1,6,7]$. The ultimate aim of dentin bonding agents is to enable proper penetration of resin monomers into demineralised collagen fibres exposed by acid etching and their subsequent sealing and protection against degradation [1]. It was reported that penetration of resin monomers into demineralised dentin with the use of etch-and-rinse adhesive systems decreases by an increment in demineralisation depth. The uttermost concentration of resin is found at the surface, and the concentration decreases in deep areas of the hybrid layer. The lowest concentration of adhesive is found at the deepest region of the demineralised area $[8,9]$. Also, to protect the hybrid layer against degradation, there must be complete infiltration and sealing of the exposed collagen mesh. It seems that bonding quality is not dependent on the length and quantity of resin tags [1]. However, there are different approaches applicable for improving the dentin bonding. For instance, Lapinska et al. [10] applied chlorhexidine prior to bonding and concluded that utilising the mentioned material before adhesive treatment may result in durability of adhesive bonding.

On the other hand, considering the existence of residual solvent and outward motion of intratubular fluid, complete penetration of monomer into the demineralised layer of dentin is not feasible. This leads to incomplete penetration of resin into hydrated collagen fibres, creating weak areas in the hybrid layer regarding degradation [8, 11]. For these reasons, recent hypotheses have focused on removing or decreasing the residual organic components and demineralised collagen network $[8,12]$.

It seems that proteoglycans play a pivotal role in the dentin extracellular matrix, due to their inherent ability in bonding to water, and have a key role in the size of interfibrillar collagen spaces $[13,14]$. The interfibrillar regions in acid-etched dentin have proteoglycans. This hydrated area has a negative charge and does not allow the permeation of large bisphenol A-glycidyl methacrylate molecules. Penetration of smaller mole- cules such as hydroxyethylmethacrylate generates a linear polymer and does not cross-link. Thus, weak areas form in the hybrid layer with high strain that eventually results in fatigue of collagen fibres. If the intertubular regions are not filled with resin, they make the hybrid layer susceptible to degradation [8].

Some studies have recommended the use of trypsin for complete elimination of proteoglycans from the etched dentin surface $[15,16]$. It seems that after surface treatment with trypsin, organic components in the lumen of tubules are eliminated and the tubules become wider. Therefore, removal of proteoglycans enhances further penetration of adhesive monomers into demineralised dentin and dentinal tubules, forming a stable interface resistant to degradation over time $[2,15]$.

The next item that has been considered is the pineapple enzyme called bromelain. It was widely used in dentistry research because it has a high solubility in water, does not contain toxic substances, and has antibacterial activity $[17,18]$. Also it was used orally or topically for wounds and burns $[19,20]$.

Bromelain has also been recommended for removal of collagen from the etched dentin surface $[2,6]$. It seems that removal of collagen from acid-etched dentin by the action of bromelain decreases the organic components of the substrate, changes the hydrophilic attributes of dentin, dilates the dentinal tubules, increases the surface energy of dentin, and subsequently improves the penetration depth of resin monomers into the substrate $[2,6]$.

Because these two deproteinised agents, bromelain and trypsin, in association with the fifth generation of bonding, have not been used in 5\% concentration and assessed in term of microleakage, this study focuses on this subject for the first time.

\section{OBJECTIVES}

The main objective of the present work is finding an appropriate chemical substance to improve the quality of the bonding process and reducing the residual organic components after etching the dentin in the clinical setting. In this regard, two procedures were followed by employing bromelain and trypsin, which are comprehensively explained in the following sections. As the null 
hypothesis, it is considered that pretreatment of etched cavity surfaces with $5 \%$ bromelain and $5 \%$ trypsin solution prior to the application of Adper Single Bond and OptiBond Solo bonding agents will have no impact on the occlusal and gingival marginal microleakage of the utilised composites.

\section{MATERIAL AND METHODS}

A total of 60 sound extracted premolars of human with no caries, previous restoration, or occlusal wear were utilised in the present in vitro experimental study. The samples had been extracted in the preceding 90 days for orthodontic purposes and immersed in a solution of $0.2 \%$ thymol. Seven days before the test, the samples were preserved in distilled water. Standard class $\mathrm{V}$ cavities, which were $3 \mathrm{~mm}$ and $2 \mathrm{~mm}$ mesiodistally and occluso-gingivally, respectively, with a depth of $1.5 \mathrm{~mm}$, were made ready in the lingual and buccal surfaces of each sample by applying a high-speed hand-piece and a diamond bur (008/835, Microdont, New York, USA) under water coolant according to ISO/ TS11405. The cavities were prepared such that the occlusal and gingival margins were $1 \mathrm{~mm}$ above and below the cementoenamel junction, respectively. The occlusal margin was bevelled by $0.5 \mathrm{~mm}$. The samples were randomly assigned to three groups $(n=20)$ with class V cavities prepared on their lingual and buccal surfaces $(n=40$ cavities). The preparation procedures of the groups are explained in the following parts of the article.

\section{CONTROL GROUP (CG)}

The etching of the enamel margin took 13 seconds, while the dentin areas were etched with $37 \%$ phosphoric acid (Gel etchant, Kerr Italia SPA, Salerno, Italy) for 15 seconds. The cavity was rinsed for 60 seconds, and the extra water was eliminated by a cotton pellet. Single Bond (3M ESPE, St. Paul, MN, USA) was used in 20 cavities on the basis of the instruction of the manufacturer. Utilising an applicator, two layers of this bonding agent were employed on the cavity walls followed by five seconds of gentle air spray (for solvent removal) and 10 seconds of curing utilising a light curing system (Bluephase, Ivoclar Vivadent, Australia). The compositions of bonding agents are represented in Table 1 . The light intensity of the system was equal to $1200 \mathrm{~mW} / \mathrm{cm}^{2}$.

Based on the instruction of the manufacturer, OptiBond Solo (Kerr Italia SPA, Salerno, Italy) was employed in the remaining 20 cavities. Using an applicator, it was used on the cavity walls, air sprayed for three seconds (for solvent removal), and light cured for 20 seconds.

Z250 composite (3M ESPE, St. Paul, MN, USA) A3 shade was used in three increments of occlusal, gingival, and middle by a spatula. Each increment was individually light cured for 20 seconds. The entire restoration was cured again for 40 seconds. According to the catalogue of this material, its volumetric shrinkage is approximately $2 \%$.

\section{BROMELAIN GROUP (BG)}

First, $5 \%$ bromelain solution was prepared by dissolving $0.5 \mathrm{~g}$ of bromelain powder (Sigma Aldrich, St. Louis, MO, USA) in $10 \mathrm{cc}$ of distilled water. After acid etching of the cavity, the bromelain solution was used on the wall of cavity for 30 seconds using an applicator, rinsed with water, which took 15 seconds, and the extra water was eliminated utilising a cotton pellet. Applying the composite and bonding agent was similar to the control group.

\section{TRYPSIN GROUP (TG)}

First, $0.5 \mathrm{~g}$ of trypsin powder (Sigma Aldrich, St. Louis, MO, USA) was dissolved in $10 \mathrm{cc}$ of water. After acid etching of the cavity, $5 \%$ Trypsin solution was used on the wall of the cavity for 30 seconds with an applicator, rinsed with water, which took 15 seconds, to keep the $\mathrm{pH}$ value in an appropriate range (approximately 7), and similarly to the previous groups - the excess water was eliminated. Applying the composite and bonding agent was similar to the control group.

The restored samples were incubated for 24 hours in $37^{\circ} \mathrm{C}$; afterwards, the samples were polished by employing coarse to fine-grit abrasive discs (Kerr Hawe SA, Bioggio, Switzerland). Subsequently, all samples were thermocycled for 5000 cycles in temperature range of $5-55^{\circ} \mathrm{C}$ with a dwell and transfer time of 30 seconds.

TABLE 1. The composition of bonding agents used in this study

\begin{tabular}{|c|c|c|}
\hline Adhesive agents & Composition & Instructions for use \\
\hline $\begin{array}{l}\text { Adper Single Bond } 2 \\
\text { (3M ESPE, St Paul, MN, USA) }\end{array}$ & $\begin{array}{l}\text { Ethyl alcohol, Bis-GMA, silane-treated silica, 2-hydroxyethyl } \\
\text { methacrylate (HEMA), glycerol 1,3-dimethacrylate, diurethane } \\
\text { dimethacrylate, copolymer of acrylic and itaconic acids }\end{array}$ & $\begin{array}{l}\text { Apply two consecutive coats of adhesive to the tooth } \\
\text { surface with gentle agitation for } 15 \mathrm{~s} \text {; gently air thin; } \\
\text { light cure for } 10 \mathrm{~s}\end{array}$ \\
\hline $\begin{array}{l}\text { OptiBond Solo } \\
\text { (Kerr SPA, Salerno, Italy) }\end{array}$ & $\begin{array}{l}\text { Bis-GMA, GPDM, GDMA, HEMA, ethyl alcohol, mono and difunctional } \\
\text { methacrylate monomers, CQ, fumed Silica, barium aluminium } \\
\text { borosilicate glass, sodium hexafluorosilicate }\end{array}$ & $\begin{array}{l}\text { Apply the adhesive and rub for } 15 \mathrm{~s} \text { and dry for } 3 \mathrm{~s} \text {. } \\
\text { Light cure for } 20 \mathrm{~s}\end{array}$ \\
\hline
\end{tabular}




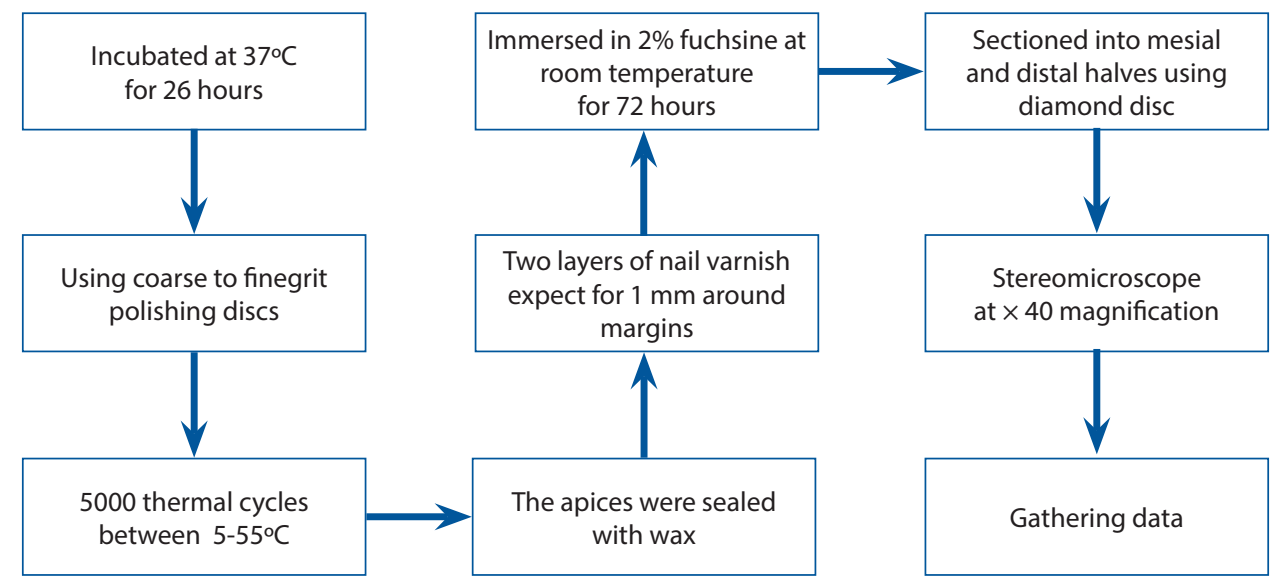

FIGURE 2. The process of preparing samples

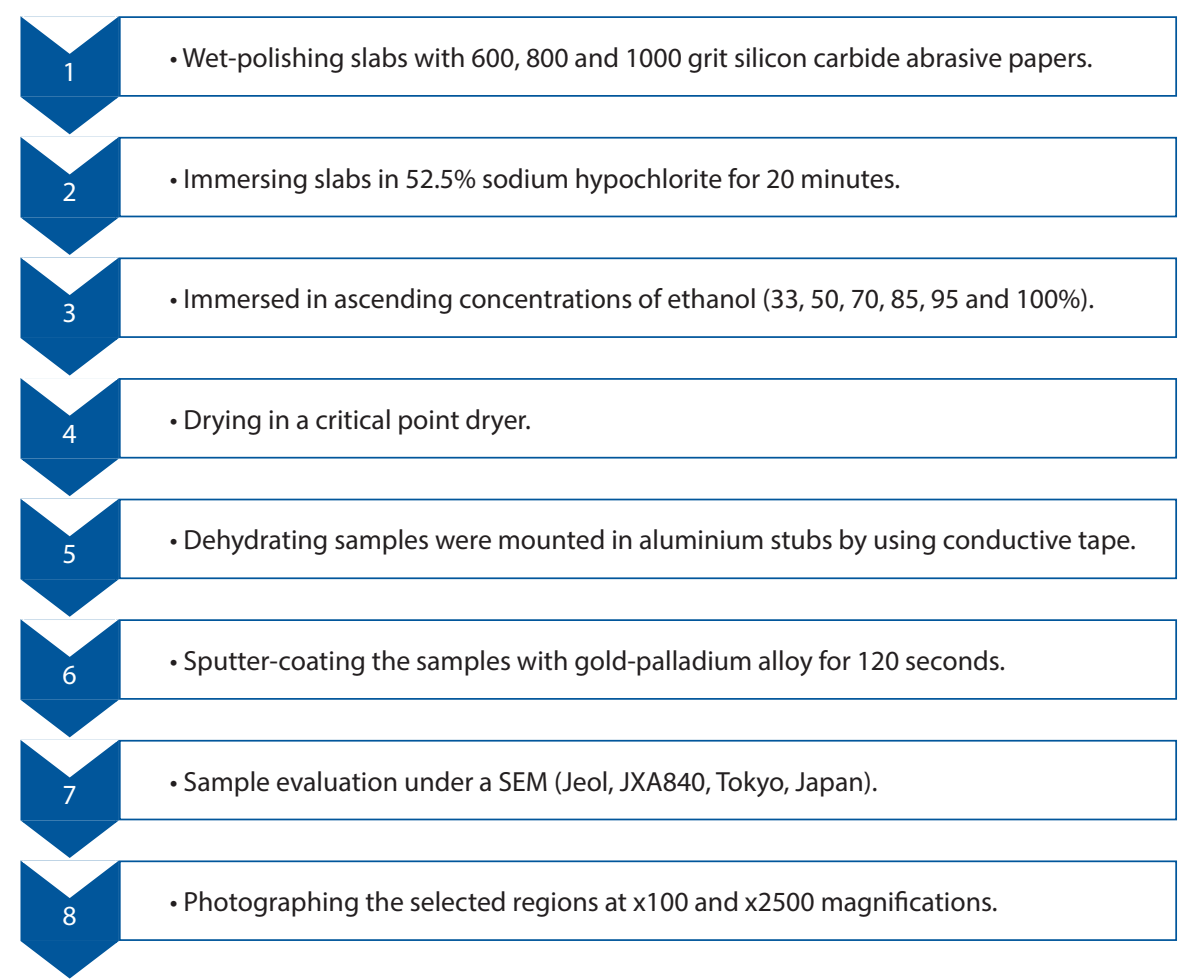

FIGURE 3. Preparing samples for SEM analysis

\section{DYE PENETRATION PROCESS}

The root apices were sealed by using stick wax, and two layers of nail varnish were used for coating the surfaces of the teeth. Subsequently, the samples were immersed in 2\% fuchsine (Merck, Darmstadt, Germany) solution at room temperature for 72 hours and were divided into distal and mesial halves by using a double-blade diamond disc with $3 \mathrm{~mm}$ thickness. The microleakage degree at the gingival and occlusal margins was determined by using stereomicroscope (ZEISS, Jena, Germany) at 40x magnification. All observations were made by one observer two times, and the higher value was announced as the microleakage degree (score). The process of preparation of the samples is depicted in Figure 2.

Scoring of microleakage at the gingival and occlusal margins was performed by utilising the scoring format as follows:

- 0 : Without any dye penetration,

- 1: Dye penetration to $1 / 2$ of the gingival or occlusal wall,

- 2: Dye penetration exceeding $1 / 2$ of the gingival or occlusal wall but not reaching the axial wall,

- 3: Dye penetration to the tooth-restoration interface reaching the axial wall.

The gathered data were investigated by utilising SPSS version 24 through descriptive statistics, Mann Whitney 
TABLE 2. Frequency and comparison of microleakage scores in the study groups

\begin{tabular}{|c|c|c|c|c|c|c|}
\hline \multirow{2}{*}{ Margin } & \multirow{2}{*}{ Study group } & \multicolumn{4}{|c|}{ Microleakage score } & \multirow{2}{*}{${ }^{*} p$-value } \\
\hline & & 0 & 1 & 2 & 3 & \\
\hline \multirow{3}{*}{ Occlusal margin } & $C G$ & 37 (92.5\%) & $3(7.5 \%)$ & 0 & 0 & \multirow{3}{*}{0.43} \\
\hline & BG & 38 (95\%) & $2(5 \%)$ & 0 & 0 & \\
\hline & TG & $35(87.5 \%)$ & $3(7.5 \%)$ & $2(5 \%)$ & 0 & \\
\hline \multirow{3}{*}{ Cervical margin } & CG & $26(65 \%)$ & $9(22.5 \%)$ & $3(7.5 \%)$ & $2(5 \%)$ & \multirow{3}{*}{0.0001} \\
\hline & $B G$ & $32(80 \%)$ & $4(8 \%)$ & $4(8 \%)$ & 0 & \\
\hline & TG & $15(37.5 \%)$ & $10(25 \%)$ & $7(17.5 \%)$ & $8(20 \%)$ & \\
\hline
\end{tabular}

*Kruskal-Wallis analysis. Similar uppercase letters indicate statistically similar means, according to the Bonferroni post hoc tests ( $p>0.008)$

test, Kruskal-Wallis test (at $p<0.05$ level of significance), and a post hoc test (at $p<0.008$ level of significance).

SEM: For micro-morphological assessment of the resin-dentin interface when using a scanning electron microscope (SEM), the pieces were wet-polished by applying silicon carbide paper discs and were then etched with $35 \%$ phosphoric acid gel for 15 seconds. After rinsing, compressed air was used to dry the samples. Afterwards, the pieces were immersed in $5.25 \%$ sodium hypochlorite solution for 20 minutes and were rinsed under running water. They were dehydrated using increasing concentrations of ethanol $(33,50,70,85,95$, and $100 \%)$. After immersion in $100 \%$ ethanol, the samples were dried in a critical point dryer. The samples, which were dehydrated in previous steps, were installed on aluminium stubs using a conductive tape (double-sided carbon tapes) (3M, Sao Paulo, SP, Brazil). While placed in a vacuum evaporator (SDC050, Balzers, Liechtenstein), they were sputter coated (bal-tec, scd, 005, Zurich, Switzerland) with gold-palladium alloy for 120 seconds. Subsequently, the samples were examined under a SEM (JEOL-JXA-840, Tokyo, Japan). Some of the chosen regions of the resin-dentin interface (two images of the centre of each quadrant) were photographed at 1000x and 2500x magnifications. The procedure of preparing the samples for SEM analysis is summarised in Figure 3.

\section{RESULTS}

The Mann-Whitney test revealed that in each group, cervical margin microleakage was significantly higher compared with occlusal margin microleakage $(p<0.05)$.

The Kruskal-Wallis test indicated no significant difference in occlusal margin microleakage among the considered groups; however, the difference in cervical margin microleakage was statistically significant $(p<0.05$, Table 2).

According to the Bonferroni correction, the significance level was set at $p<0.008$ Post hoc test indicates a significant increase in microleakage of cervical margins in TG (Table 3).

According to the obtained data, the hybrid layer is thicker in BG compared with CG. Moreover, the resin
TABLE 3. Pairwise comparison between cervical margins of studied groups

\begin{tabular}{|l|c|c|}
\hline \multicolumn{1}{|c|}{ Study group } & CG & BG \\
\hline BG & 0.154 & - \\
\hline TG & $0.005^{*}$ & $0.0001^{*}$ \\
\hline
\end{tabular}

Significant level determinate 0.008 by Post hoc analysis. ${ }^{*}$ shows significant difference between groups

tag orifice is larger and is conical in this group. Furthermore, spherical residues are observed on the resin tags, which are due to the slight penetration of resin into the accessory dentinal canals. In TG, the resin tag orifice is narrower compared with CG and does not have adequate length and is not uniform. Moreover, in some areas, hybrid layer disintegration is observed.

\section{DISCUSSION}

The most significant structure that influences the micromechanical retention of resin restorations is hybrid. It is also responsible for the sealing of dentin. Nonetheless, the hybrid layer is the most common failure site at the adhesive interface [21]. It has been reported that reduction in protein content of the acid-etched dentin by the activity of proteolysis agents can increase the penetration depth of resin monomers into the demineralised dentin collagen network and result in more stable hybrid layer formation at the dentin-composite interface. Materials used for this purpose are often toxic and volatile [22] or should be applied for long periods of time, which makes them unsuitable for use in the clinical setting [23-25]. The materials used for protecting the resin-dentin interface should have suitable clinical application time and low cytotoxicity [26]. Thus, considering the previous studies $[2,15]$ in the current study, $5 \%$ bromelain and $5 \%$ trypsin were applied on dentin surfaces for 30 seconds.

The solvent type in the bonding system mixture is a principle parameter determining depth of etchand-rinse adhesive system penetration into the dentin surface [4]. The bond strength improves by increasing 

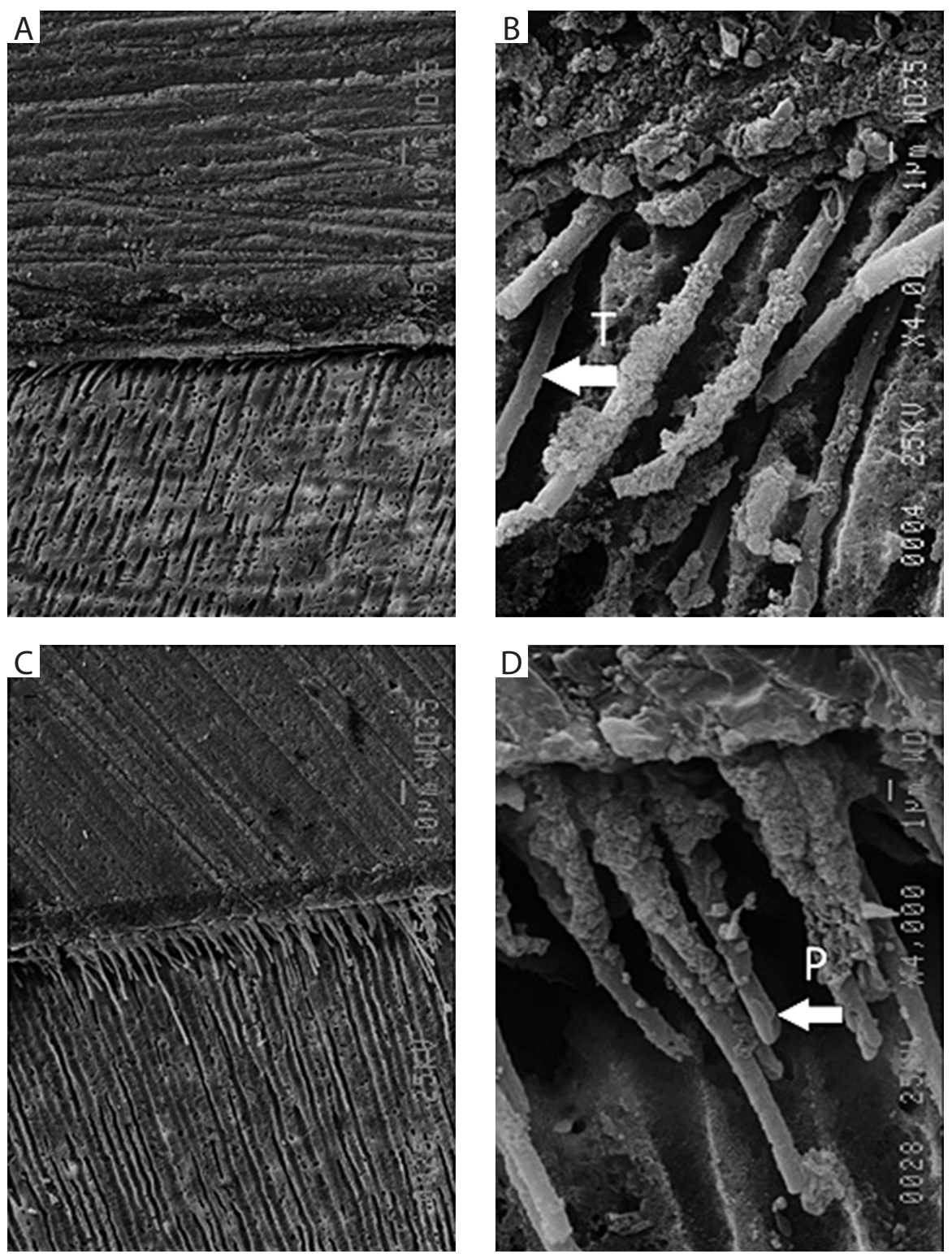

FIGURE 4. Selected SEM micrograph of the resin-dentin interface in the control groups: A, B) Single Bond, 500× and 4000× magnifications; C, D) OptiBond, 500× and 4000× magnifications. Conical resin tags with rough surfaces ( $\mathrm{T}$ ) are seen. A small number of accessory canals are filled with resin (P)

the solvent penetration depth into the etched dentin and enhancing the chemical integrity of the dentin and resin [12]. In the current research, adhesive systems that used ethanol solvent were utilised. It is necessary to mention that the two systems are different in terms of the amount of solvent and other components of resin in their chemical content.

Surface treatment of etched dentin with trypsin for 30 seconds in this study did not cause a significant change in the occlusal margin microleakage but increased the microleakage at the cervical margins. Therefore, the null hypothesis of the present study regarding the use of trypsin was rejected. This result was in line with the findings of some studies $[16,27]$ but in contrast to those of some other studies [15].
Some studies have reported that application of $1 \mathrm{mg} / \mathrm{ml}$ trypsin solution for 48 hours on acid-etched dentin eliminates non-collagenous proteins and proteoglycans from dentin and increases the thickness of the hybrid layer and its strength $[15,16]$. Therefore, the role of non-collagenous proteins in the bonding mechanism has recently been highlighted. Electrophoretic assessment with the SDS-PAGE method showed extrusion of non-collagenous molecules, including proteoglycans, from the tissue following the use of trypsin [16]. Some studies have shown that the increased microporosities of dentin following treatment with trypsin are due to the removal of the interfibrillar structure $[27,28]$. Because water plays key role in preserving the interfibrillar collagen space after etching demineralised dentin, it seems that 

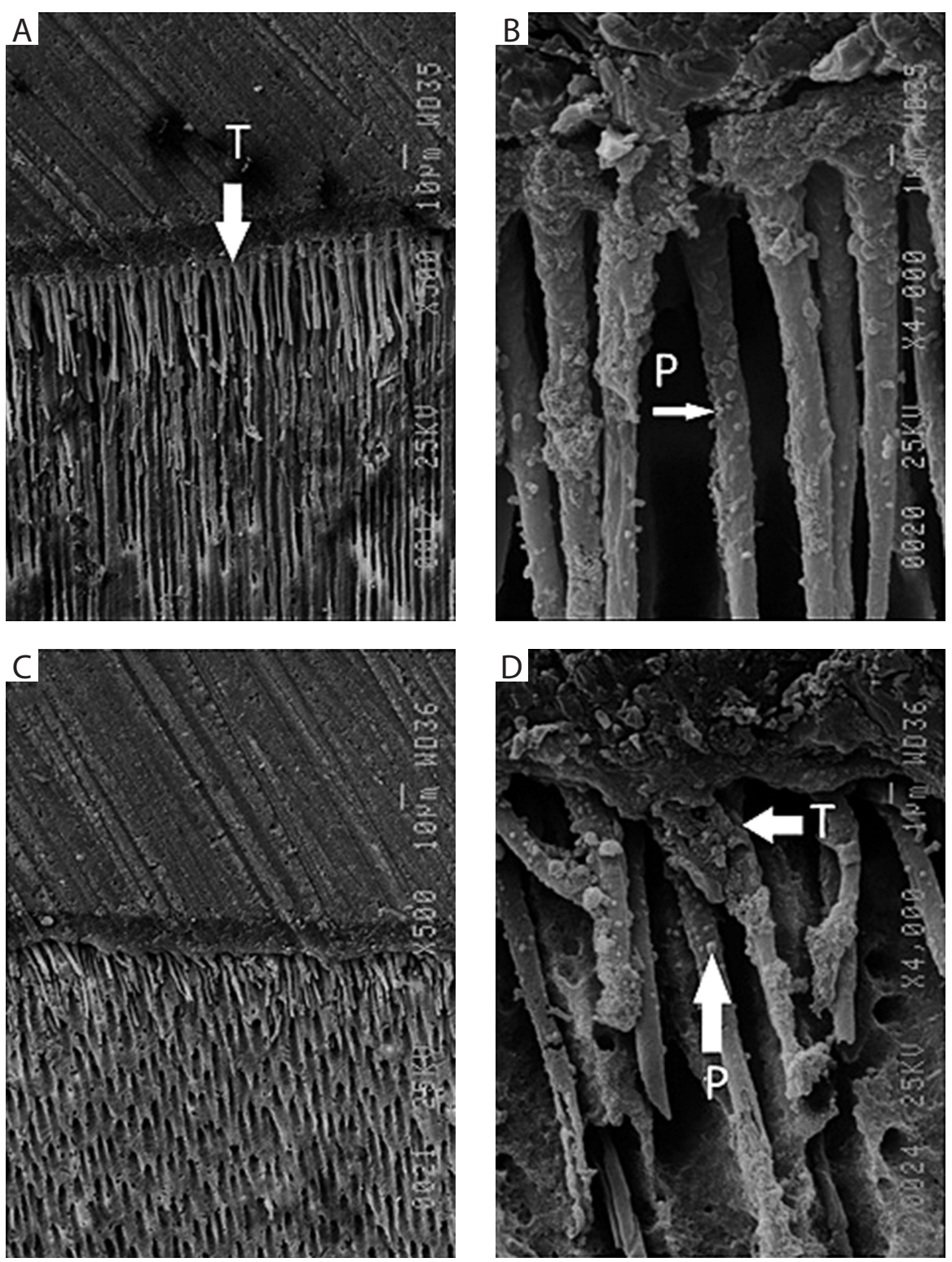

FIGURE 5. Selected SEM micrograph of the resin-dentin interface following the application of bromelain: A, B) Single Bond at 500x and 4000x magnifications; C, D) OptiBond at 500× and 4000× magnifications. The hybrid layer in this group is thicker than that in the other two groups. The orifice of the resin tags is larger and they are coni$\mathrm{cal}(\mathrm{T})$ in this group. Spherical residues are seen on the resin tags formed as a result of slight penetration of resin into the accessory dentinal canals $(P)$

glycosaminoglycans, due to their hydrophilicity, significantly influence the preservation of the collagen fibres by filling the interfibrillar spaces in a hydrated status. Therefore, by removing the glycosaminoglycan chains with trypsin, the capacity of collagen fibres to serve as a suitable scaffold for monomer penetration decreases, which further decreases the penetration depth of hydrophilic monomers, impairs the hybrid layer formation, and decreases the bond strength [2].

On the other hand, topographic changes and vertical grooves in collagen fibres and dissolution of some compounds at the surface and centre of larger fibres can also change the mechanical features of demineralised dentin, such as its hardness and creep [29]. Therefore, it seems that trypsin can degrade the weak bonds between micro-fibrils $[15,16]$.

Because the significance of etch-and-rinse systems and first generations of self-etch adhesives is due to simple diffusion of monomer molecules into the collagen structure in the absence of functional monomers, the mechanism of diffusion into the collagen structure is very restricted. Dissolution of collagen bundles occurs following the elimination of superficial proteoglycans by trypsin. Following an increase in dentin porosity, diffusion of bonding agent into the matrix increases $[15,29]$. 

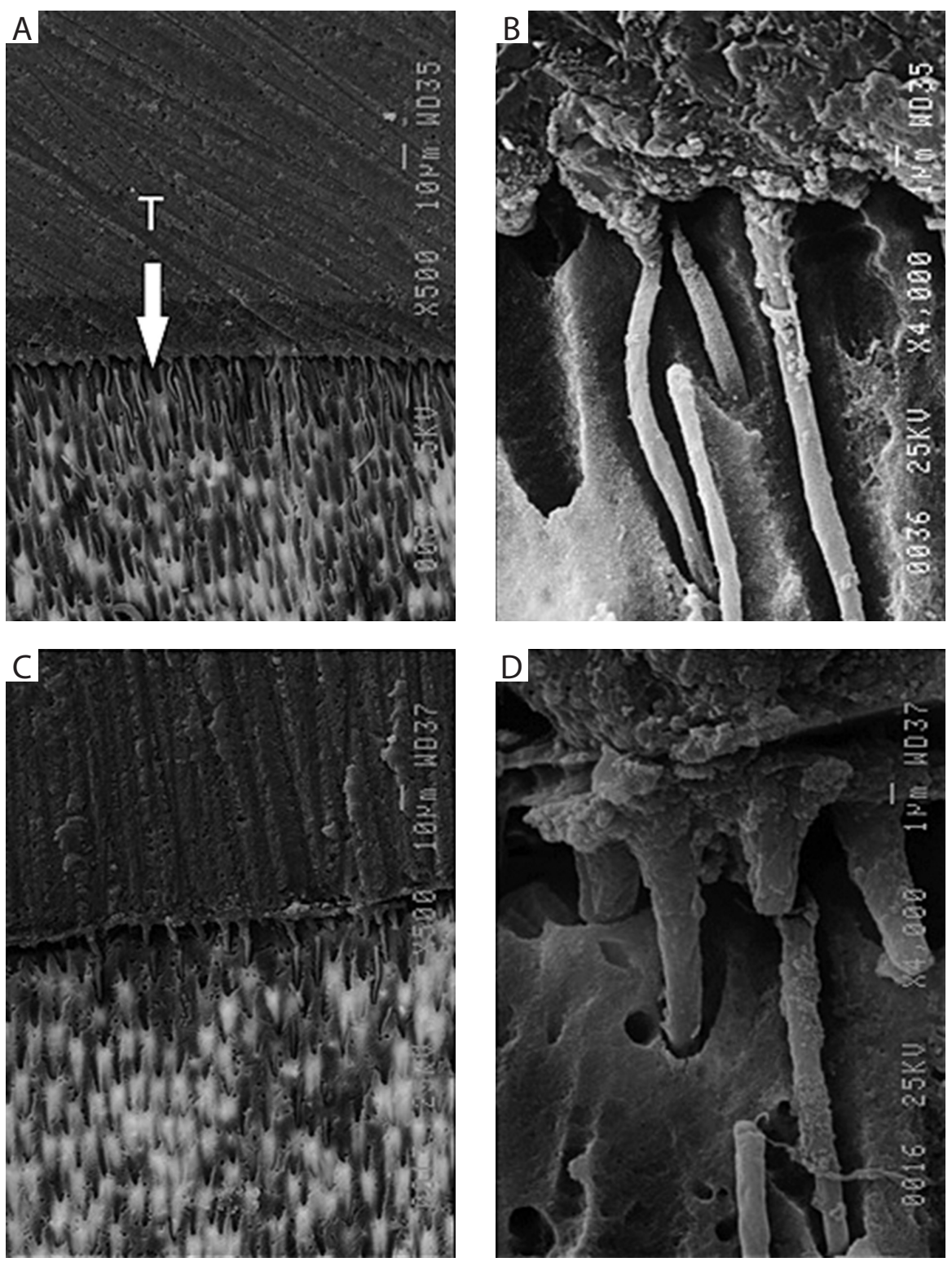

FIGURE 6. Selected SEM micrograph of the resin-dentin interface following the application of trypsin: A) Single Bond at 500× magnification, B) Single Bond at 4000× magnification, C) OptiBond at 500× magnification; D) OptiBond at 4000x magnification. The orifice of the resin tags is narrower in this group, they do not have adequate length, and they are not uniform. Also, disintegration of the hybrid layer is seen in some areas (T)

In the current study, the microleakage at the dentinal margin of restorations in the trypsin group was significantly higher compared with the other groups. This result may be due to the fact that $5 \%$ trypsin removes the interfibrillar structure and degrades the 3D structure of the collagen network. As a result, hydrophilic monomers are eliminated or their penetration into etched intertubular dentin decreases. Eventually, based on the SEM micrographs of the resin-dentin interface in studied groups, the proper hybrid layer formation is impaired in preparation of the dentin surface with $5 \%$ trypsin (Figure 6). Reduction in the amount of twisted collagen micro-fibrils [16], polymerisation shrinkage, and marginal gap and the location of the gingival margin (in terms of presence of greater moisture and organic compounds in this area) [28] are among the main reasons for impairment of the bonding mechanism and increased microleakage at the dentinal margin in this group.

The current study showed that although microleakage at the occlusal and cervical margins of BG was decreased, it was not significantly different in comparison with the CG. Thus, the null hypothesis was confirmed. In this regard, Sharafeddin and Haghbin [20] found no difference in SBS by using 3\% bromelain. Despite the fact that some parameters such as cavity preparation and the percentage of bromelain enzymes that were used in their study were different from the current research, our results are favourable. Sharafeddin and Safari [19] 
in 2019 reported that using 6\% bromelain enzyme before acid-etching had no impact on SBS of composite to superficial dentin; however, it could be related to the different steps of using the enzymes within our study. This finding does not endorse previous studies $[2,6]$. Dayem et al. [6] demonstrated that utilisation of bromelain on etched dentin significantly reduced microleakage, which was attributed to the ability of bromelain to take collagen from the etched dentin. In another study [2], a similar observation was reported. It was observed that bromelain enhanced the dentin substrate permeability by removing collagen from the acid-etched surface and resulted in broadening of dentinal tubules close to the external surface, increased the energy of the surface, and enhanced the penetration and infusion of monomers in dentin [2]. Hydroxyapatite has higher surface energy compared with collagen. Thus, depletion of collagen in the etched dentin reduces the organic compounds and enhances the energy of the surface, changing the hydrophilic nature of dentin; therefore, more appropriate adhesive monomer penetration into dentin is provided [6]. SEM micrographs in the current study showed that in BG (Figure 5), the hybrid layer was thicker and resin tags had a larger orifice and were conical in shape with little resin penetration into accessory dentinal canals. These changes occur in the etched dentin following the application of bromelain. However, no significant difference was noted in microleakage at the dentin margin between the BG and CG. Some other influential factors may interfere with the outcome of this type of surface treatment, including the duration and percentage of bromelain application. In the previous research $[2,16]$, pure bromelain was applied on dentin surfaces for one minute, which was different from the application protocol in our study.

On the other hand, following acid etching, matrix metalloproteinases in the matrix components become activated, resulting in degradation of collagen fibres and their elimination from the hybrid layer. The space created by collagen depletion is filled with water. Thus, increased degradation of the bond due to hydrolysis and subsequent microleakage are expected to occur [29]. SEM assessment of the hybrid layer in this group in comparison with the CG (as shown in Figures 4 and 5) revealed that treatment of etched dentin with $5 \%$ bromelain for 30 seconds resulted in further penetration of adhesive into the hybrid layer. However, no significant reduction occurred in marginal microleakage. Considering the hydrophilicity of bromelain, it can probably server as a factor to retain water in the substrate and as such affect the degree of conversion. Therefore, bromelain decreases the microleakage but also serves as a hydrophilic agent in the area and increases the microleakage. Further research is needed to optimise the effective concentration of bromelain enzyme for reduction of microleakage at the dentin margin of restorations bonded with etch-and-rinse adhesive systems.
To sum up, it must be indicated that the null hypothesis is accepted for BG, while it is rejected for TG.

\section{CONCLUSIONS}

In the present work, microleakage of restorations in cases of employing bromelain and trypsin were evaluated. According to the results of SEM, a thicker hybrid layer was observed for the cases in which bromelain was applied. Moreover, a narrower resin tag orifice existed for the trypsin group compared with the control group. Based on the Kruskal-Wallis test, it was found that there was no significant difference in occlusal margin microleakage among the three considered groups; however, the difference in cervical margin microleakage was significantly higher in the case of utilising trypsin.

\section{CONFLICT OF INTEREST}

The authors declare no potential conflicts of interest with respect to the research, authorship, and/or publication of this article.

\section{References}

1. Matos AB, Trevelin LT, da Silva BTF, Francisconi-Dos-Rios LF, Siriani LK, Cardoso MV. Bonding efficiency and durability: current possibilities. Braz Oral Res 2017; 31: 3-22.

2. Chauhan K, Basavanna RS, Shivanna V. Effect of bromelain enzyme for dentin deproteinization on bond strength of adhesive system. J Conserv Dent 2015; 18: 360-363.

3. Goldstein RE, Lamba S, Lawson NC, Beck P, Oster RA, Burgess JO. Microleakage around class $\mathrm{V}$ composite restorations after ultrasonic scaling and sonic toothbrushing around their margin. J Esthet Restor Dent 2017; 29: 41-48.

4. Zheng P, Chen H. Evaluate the effect of different mmps inhibitors on adhesive physical properties of dental adhesives, bond strength and mmp substarte activity. Sci Rep 2017; 7. DOI: 10.1038/s41598017-04340-1.

5. Xu C, Wang Y. Cross-linked demineralized dentin maintains its mechanical stability when challenged by bacterial collagenase. J Biomed Mater Res Part B Appl Biomater 2011; 96B: 242-248.

6. Dayem R, Tameesh M. A new concept in hybridization: bromelain enzyme for deproteinizing dentin before application of adhesive system. Contemp Clin Dent 2013; 4: 421.

7. Scheffel DLS, Hebling J, Scheffel RH, et al. Inactivation of matrix-bound matrix metalloproteinases by cross-linking agents in acid-etched dentin. Oper Dent 2014; 39: 152-158.

8. Liu Y, Tjäderhane L, Breschi L, et al. Limitations in bonding to dentin and experimental strategies to prevent bond degradation. J Dent Res 2011; 90: 953-968.

9. Zhang SCh, Kern M. The role of host-derived dentinal matrix metalloproteinases in reducing dentin bonding of resin adhesives. Int J Oral Sci 2009; 1: 163-176.

10. Lapinska B, Klimek L, Sokolowski J, Lukomska-Szymanska M. Dentine surface morphology after chlorhexidine application-SEM study. Polymers (Basel) 2018; 10: 905.

11. Pashley DH, Tay FR, Breschi L, et al. State of the art etch-andrinse adhesives. Dent Mater 2011; 27: 1-16.

12. Dayem RN. Assessment of the penetration depth of dental adhesives through deproteinized acid-etched dentin using neodymium: yttrium-aluminum-garnet laser and sodium hypochlorite. Lasers Med Sci 2010; 25: 17-24. 
13. Breschi L, Lopes M, Gobbi P, Mazzotti G, Falconi M, Perdigão J. Dentin proteoglycans: an immunocytochemical FEISEM study. J Biomed Mater Res 2002; 61: 40-46.

14. de Mattos Pimenta Vidal C, Leme-Kraus AA, Rahman M, Farina AP, Bedran-Russo AK. Role of proteoglycans on the biochemical and biomechanical properties of dentin organic matrix. Arch Oral Biol 2017; 82: 203-208.

15. Lu S, Zhao SJ, Gao Y, Sun Y, Li X, Chen JH. Proteoglycans affect monomer infiltration in the etch-and-rinse bonding technique. Dent Mater 2014; 30: e289-e299.

16. Bertassoni LE, Swain M V. Removal of dentin non-collagenous structures results in the unraveling of microfibril bundles in collagen type I. Connect Tissue Res 2017; 58: 414-423.

17. Liliany D, Widyarman A, Erfan E, Sudiono J, Djamil M. Enzymatic activity of bromelain isolated pineapple (Ananas comosus) hump and its antibacterial effect on Enterococcus faecalis. Sci Dent J 2018; 2: 39.

18. Oliveira JBS, Sarlo RS, Bresciani E, Caneppele TMF. Whitening efficacy of whitening mouth rinses used alone or in conjunction with carbamide peroxide home whitening. Oper Dent 2017; 42: 319-326.

19. Sharafeddin F, Safari M. Effect of papain and bromelain enzymes on shear bond strength of composite to superficial dentin in different adhesive systems. J Contemp Dent Pract 2019; 20: 1077-1081.

20. Sharafeddin F, Haghbin N. Comparison of bromelain enzyme, sodium hypochlorite, and titanium tetrafluoride on shear bond strength of restorative composite to dentin: an in vitro study. J Dent 2019; 20: 264.

21. Scheffel DLS, Hebling J, Scheffel RH, et al. Stabilization of dentin matrix after cross-linking treatments, in vitro. Dent Mater 2014; 30: 227-233.

22. Han B, Jaurequi J, Tang BW, Nimni ME. Proanthocyanidin: a natural crosslinking reagent for stabilizing collagen matrices. J Biomed Mater Res 2003; 65A: 118-124.

23. Al-Ammar A, Drummond JL, Bedran-Russo AK. The use of collagen cross-linking agents to enhance dentin bond strength. J Biomed Mater Res Part B Appl Biomater 2009; 91B: 419-424.

24. Castellan CS, Bedran-Russo AK, Karol S, Pereira PNR. Long-term stability of dentin matrix following treatment with various natural collagen cross-linkers. J Mech Behav Biomed Mater 2011; 4: 1343-1350.

25. Macedo GV, Yamauchi M, Bedran-Russo AK. Effects of chemical cross-linkers on caries-affected dentin bonding. J Dent Res 2009; 88: 1096-1100.

26. Marshall SJ, Bayne SC, Baier R, Tomsia AP, Marshall GW. A review of adhesion science. Dent Mater 2010; 26. DOI: 10.1016/j.dental. 2009.11.157.

27. Bedran-Russo AKB, Pereira PNR, Duarte WR, Okuyama K, Yamauchi M. Removal of dentin matrix proteoglycans by trypsin digestion and its effect on dentin bonding. J Biomed Mater Res Part B Appl Biomater 2008; 85B: 261-266.

28. Savadi Oskoee S, Alizadeh Oskoee P, Jafari Navimipour E, et al. Comparison of the effect of Nd:YAG and diode lasers and photodynamic therapy on microleakage of class $\mathrm{V}$ composite resin restorations. J Dent Res Dent Clin Dent Prospects 2013; 7: 74-80.

29. Hass V, De Paula AM, Parreiras S, et al. Degradation of dentin-bonded interfaces treated with collagen cross-linking agents in a cariogenic oral environment: an in situ study. J Dent 2016; 49: 60-67. 